\title{
Relationship between Compressive Strength of Geo-polymers and Pre-curing Conditions
}

\author{
Hyunjung Kim, Yooteak Kim ${ }^{1, *}$ \\ Industry University Cooperation Foundation, ${ }^{1}$ Department of Materials Engineering, Kyonggi University, Suwon 443-760, Korea
}

*Correspondence to:

$\operatorname{Kim} Y$,

Tel: +82-31-249-9765

Fax: $+82-31-249-9774$

E-mail: ytkim@kyonggi.ac.kr

Received December 16, 2013

Revised December 26, 2013

Accepted December 26, 2013

\begin{abstract}
Meta-kaolin (MK) and blast furnace slag (BS) were used as raw materials with $\mathrm{NaOH}$ and sodium silicate as alkali activators for making geo-polymers. The compressive strength with respect to the various pre-curing conditions was investigated. In order to improve the recycling rate of $\mathrm{BS}$ while still obtaining high compressive strength of the geo-polymers, it was necessary to provide additional $\mathrm{CaO}$ to the $\mathrm{MK}$ by adding $\mathrm{BS}$. The specimens containing greater amounts of BS can be applied to fields that require high initial compressive strength. Alkali activator(s) are inevitably required to make geo-polymers useful. High temperature pre-curing plays an important role in improving compressive strength in geo-polymers at the early stage of curing. On the other hand, long-term curing produced little to no positive effects and may have even worsened the compressive strength of the geo-polymers because of micro-structural defects through volume expansion by high temperature pre-curing. Therefore, a pre-curing process at a medium range temperature of $50^{\circ} \mathrm{C}$ is recommended because a continuous increase in compressive strength during the entire curing period as well as good compressive strength at the early stages can be obtained.
\end{abstract}

Key Words: Geo-polymer, Meta-kaolin, Blast furnace slag, Alkali activator, Compressive strength

\section{INTRODUCTION}

All countries of the world concentrate various efforts on reducing the generation rate of carbon dioxide, which contributes to many natural disasters because of global warming (Temuujin \& van Riessen, 2009).

Many environmentally friendly efforts and studies on new materials replacing cement have been made to reduce carbon dioxide by considering atmospheric changes because of future industries.

Nowadays limestone powder and blast furnace slag (BS) are widely used in concrete as blended materials in cement. The replacement or Portland cement by BS can lower the cost and enhance the greenness of concrete, since the production of these two materials needs less energy and causes less $\mathrm{CO}$ emission than Portland cement. Moreover, the use of BS improves the properties of fresh and gardened concrete, such as workability and durability (Zhou et al., 2010).

Mixing a viscous ceramic powder containing $\mathrm{Al}$ and $\mathrm{Si}$ in a strong alkaline solution and polymerizing produces an alkaline alumino-silicate geo-polymer. Geo-polymers provide superior thermal resistance, adiabaticity, acid resistance, low contraction, freezing and thawing resistance, corrosion and weathering resistivity, etc. In addition, compaction and water tightness is high; and fast coagulation is possible while adhesiveness is excellent (Komnitas \& Zaharaki, 2007; Zhao et al., 2007; Provis et al., 2009).

Structural evolution in pastes produced from alkali silicateactivated meta-kaolin (MK)/BS blends is assessed. In the initial period of the reaction, the addition of MK leads to an increase in the total setting time, reduces the heat release, and affects the reaction mechanism by introduction of a large quantity of additional $\mathrm{Al}$ (Bernal et al., 2011).

Therefore, the main purpose of this study is to develop geo-

This research was supported by the Public Welfare \& Safety Research Program through the National Research Foundation of Korea (NRF) funded by the Ministry of Science, ICT \& Future Planning (Grant number: 2013M3A2A1067519).

@ This is an open-access article distributed under the terms of the Creative Commons Attribution Non-Commercial License (http://creativecommons.org/licenses/by-nc/3.0) which permits unrestricted noncommercial use, distribution, and reproduction in any medium, provided the original work is properly cited.

Copyrights @ 2013 by Korean Society of Microscopy 
polymers in which an unfired inorganic binder MK is mixed with a cement substitute material including recycled BS, which is a by-product from the steel industry, $\mathrm{NaOH}$, and silicate glass as alkali activators. The relationship between the compressive strength and pre-curing conditions was investigated to obtain the basis data for making high performance geo-polymers.

\section{MATERIALS AND METHODS}

\section{Materials}

\section{Meta-kaolin}

The MK (MetaKaolin; Nycon Materials, Seoul, Korea) was manufactured with kaolin in halloysite by a special treatment, which dehydrates the kaolin by removing coordinated water and water between the layers in order to activate the sample (amorphization).

The material is fired at high temperatures and then quenched, which preserves the crystallization energy of the kaolin and changes it to a vitreous state. This is a high-energy state in
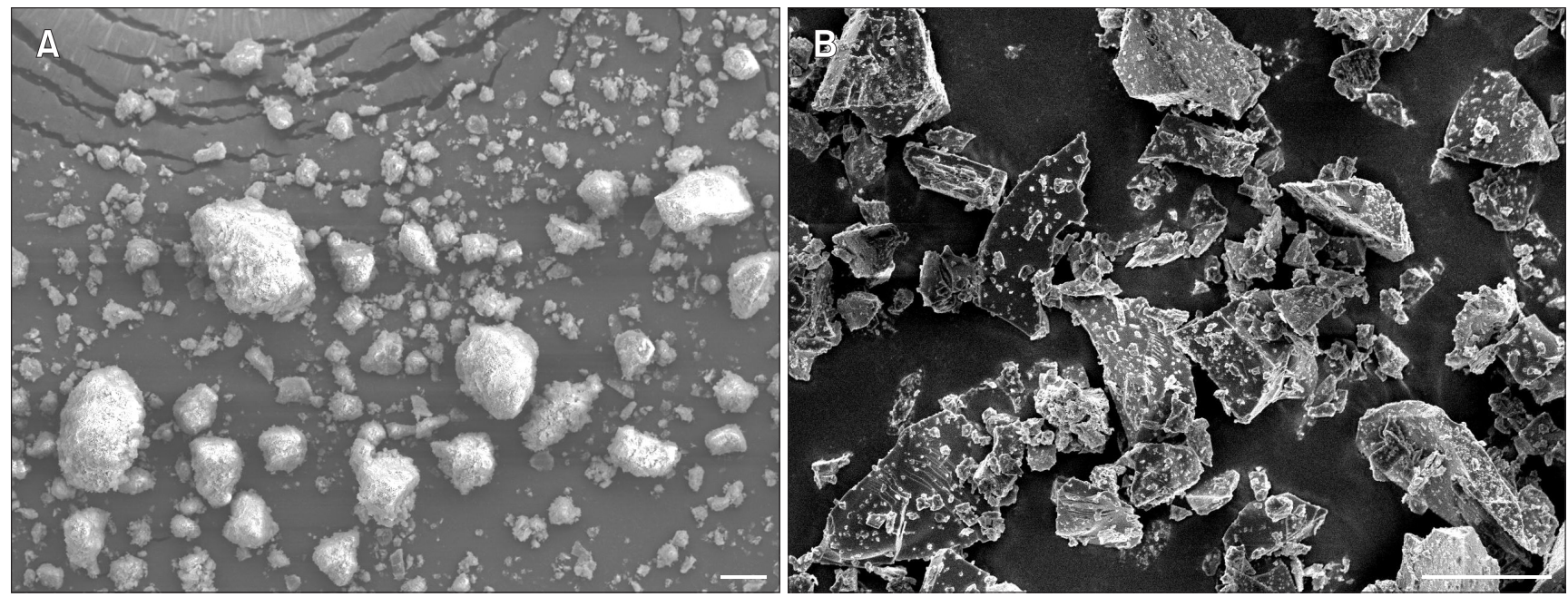

Fig. 1. Scanning electron microscopy micrographs of raw materials. (A) Meta-kaolin. (B) Blast furnace slag. Scale bars=10 $\mu$ m.

A

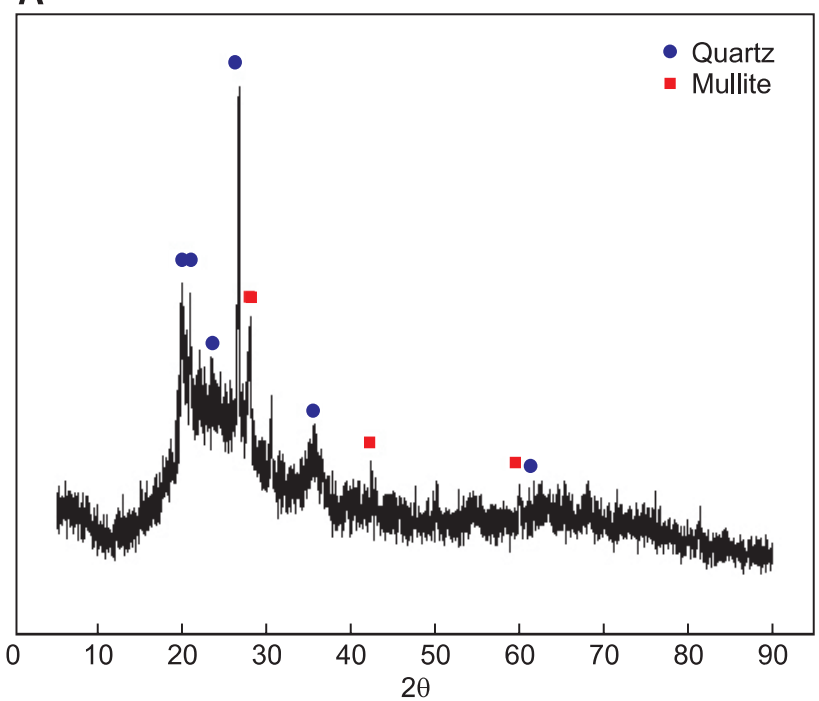

B

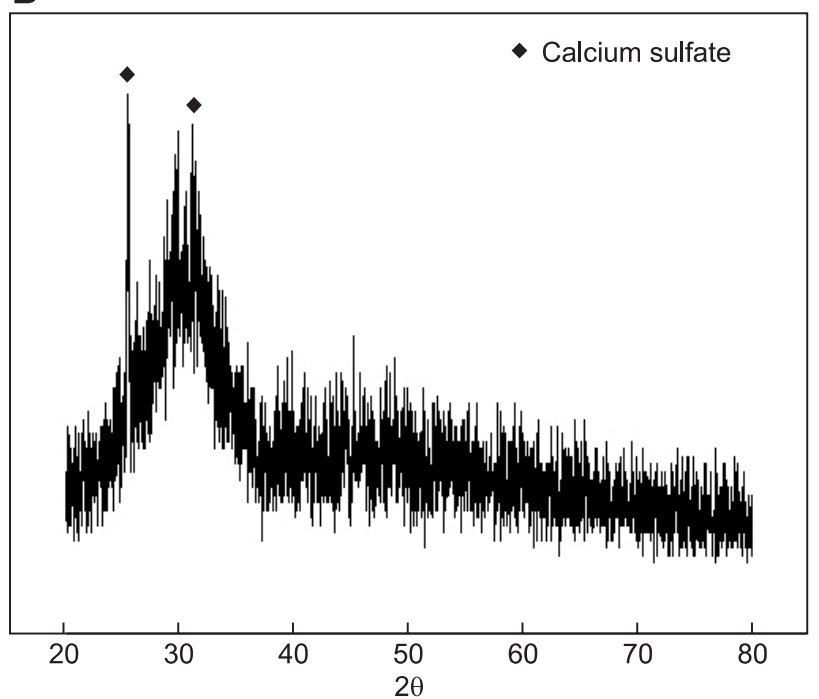

Fig. 2. X-ray diffraction patterns of raw materials. (A) Meta-kaolin. (B) Blast furnace slag. 
which the reactivity is maximized. A hydration reaction seldom occurs with the contact of water alone; however, the material will be hardened under specific hydrate conditions (with alkali activators). The activated kaolin follows a Pozzolan mechanism of reaction with $\mathrm{Ca}(\mathrm{OH})_{2}$ and gains latent hydrate hardening properties. The MK used in this study was manufactured by a domestic $\mathrm{N}$ company and its microstructure is shown in Fig. 1A. As shown in Fig. 1, the MK is comprised of round particles with a wide distribution of particles shapes and sizes. The X-ray diffraction (XRD) pattern of the MK is shown in Fig. 2A and the chemical composition is shown in Table 1.

\section{Blast furnace slag}

The BS (Blast Furnace Slag; Hankook Slag \& Materials, Incheon, Korea) is a by-product from the ion industry; it is one of the most commonly used additions in the cement industry. According to the manufacturing process, BS is a quite variable material due to the variability of its chemical composition. Offering latent hydraulic properties when mixed with clinker cement, hydration of slag is directly related to its hydraulicity: the dissolution of slag glass fraction is ensured by hydroxyl ions $\left(\mathrm{OH}^{-}\right)$resulting from the hydrolysis of Portlandite $\mathrm{Ca}(\mathrm{OH})_{2}$ produced by the hydration of clinker (Regourd, 1995; Glasser, 1996). Table 1 shows the chemical compositions of BS. The particle shapes of the BS powder are mostly irregular polygons as shown in Fig. $1 \mathrm{~B}$ and the particle distribution is narrower than that of MK. It is expected that a hydration reaction such as cementing would occur because
BS powder has not only high contents of $\mathrm{SiO}_{2}$ and $\mathrm{Al}_{2} \mathrm{O}_{3}$ (about $50 \mathrm{wt} \%$ ) but also has a high content of $\mathrm{CaO}(43 \mathrm{wt} \%)$. The XRD pattern of the BS is shown in Fig. 2B, which is characteristic of amorphous material.

\section{Alkali activator}

As the concentration of $\mathrm{OH}^{-}$is high, the $\mathrm{SiO}_{2}-\mathrm{Al}_{2} \mathrm{O}_{3}$ glassy bonding is quickly decomposed and a large amount of reactive ions is produced. When the alkali ion density is high, the alkali activator accelerates the reactant disintegration and a geo-polymer with durability and high strength can be manufactured. Sodium hydroxide $(\mathrm{NaOH}$, above $97 \%$ purity; Daejung Chemicals \& Metals, Siheung, Korea) and silicate glass (sodium silicate, $\mathrm{NaSiO}_{3}$; Deajung) No. 3 were used as alkali activators in order to promote the hardening reaction.

\section{Method}

A five-liter mortar mixer was used for mixing raw materials and activators to make the mixture of geo-polymers. The water, $\mathrm{NaOH}$, and sodium silicate were inserted in the mixer, followed by the BS and MK.

The slurry was filled in a cube mold $(50 \times 50 \times 50 \mathrm{~mm})$ and then cured for a certain time according to the pre-curing or curing conditions to sustain the shape of the specimen before the compressive strength test (Digital Type Motorized CompTester; Jeil Precision, Seoul, Korea). Specimens were sealed in a vinyl envelop after de-molding to maintain humidity during the high temperature pre-curing process. Pre-curing was done in an electric oven at room temperature $\left(25^{\circ} \mathrm{C}\right), 50^{\circ} \mathrm{C}$, and

Table 1. Chemical composition of meta-kaolin and blast furnace slag

\begin{tabular}{clllclcc}
\hline \multirow{2}{*}{ Material } & \multicolumn{7}{c}{ Chemical composition (wt\%) } \\
\cline { 2 - 8 } & $\mathrm{SiO}_{2}$ & $\mathrm{Al}_{2} \mathrm{O}_{3}$ & $\mathrm{Fe}_{2} \mathrm{O}_{3}$ & $\mathrm{CaO}$ & $\mathrm{MgO}$ & $\mathrm{SO}_{3}$ & $\mathrm{~K}_{2} \mathrm{O}+\mathrm{Na}_{2} \mathrm{O}$ \\
\hline $\mathrm{MK}$ & 52 & 40 & 3 & 1.2 & 0.5 & 0 & 1 \\
$\mathrm{BS}$ & 37.12 & 12.84 & 0.19 & 43.13 & 5.38 & 0 & 0.5 \\
\hline
\end{tabular}

MK, meta-kaolin; BS, blast furnace slag.

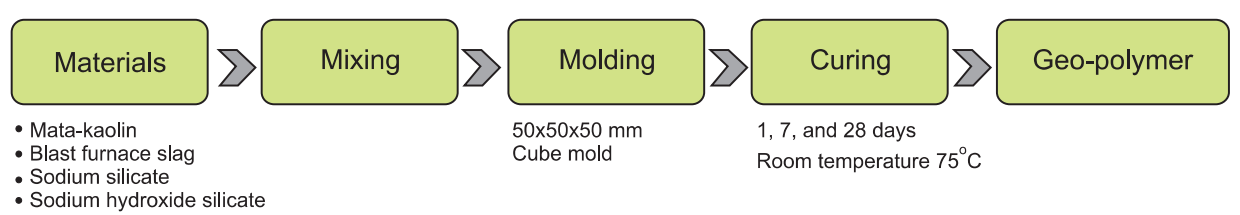

Fig. 3. Manufacturing process of geopolymer specimens.

Table 2. Mixture ratios of raw materials and alkali activators

\begin{tabular}{lccccccc}
\hline Sample & MK:BS & NaOH $(\mathrm{M})$ & Liquid/solid & Water glass (wt\%) & Water (wt\%) & Pre-curing condition & Curing condition \\
\hline Raw I & $100: 0$ & 19.48 & 0.58 & 33.33 & 66.67 & No pre-curing & Room temperature \\
Raw II & $50: 50$ & - & 0.55 & - & - & - & - \\
Raw III & $0: 100$ & - & 0.55 & - & - & - & - \\
\hline
\end{tabular}




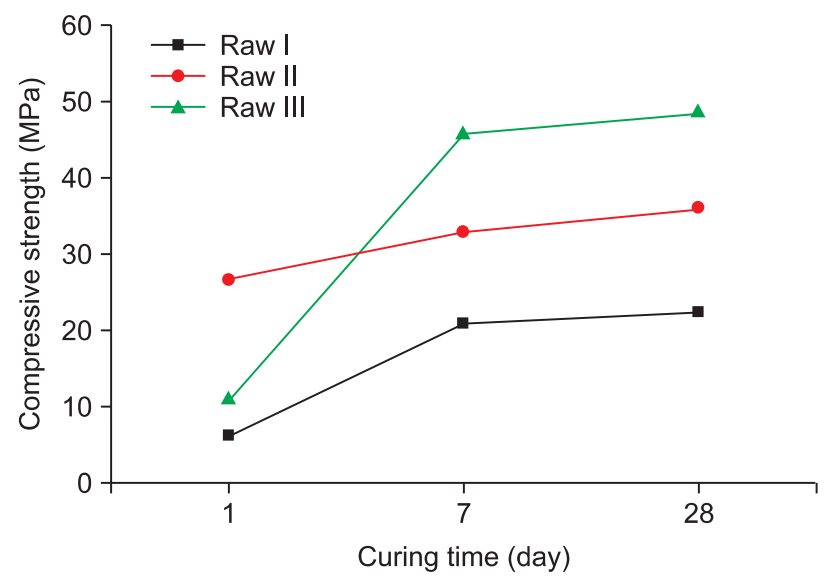

Fig. 4. Effect of mixture ratio of meta-kaolin and blast furnace slag on the compressive strength of the geo-polymers. Raw I (MK:BS=100:0), Raw II (MK:BS=50:50), and Raw III (MK:BS=0:100). $75^{\circ} \mathrm{C}$. After the pre-curing process, ordinary curing was done on all specimens for 1 day, 7 days, and 28 days.

The experimental procedure is summarized in Fig. 3 .

\section{RESULTS AND DISCUSSION}

\section{Effect of Mixing Ratio}

The amount of alkali activator was fixed and the liquid/solid ratio was maintained between 0.55 to 0.58 . The mixing ratios of the powder raw materials are given in Table 2. Fig. 4 shows the effect of the mixing ratio of MK:BS on compressive strength with room temperature curing. The compressive strength of Raw I (MK:BS=100:0) was 6.11 MPa after 1 day, which shows that the condensation by polymerization of the MK was difficult at room temperature with alkali activators. In Raw II (MK:BS=50:50) the compressive strength was 26.41 $\mathrm{MPa}$ after 1 day, and increased to $35.88 \mathrm{MPa}$ after 28 days. There exist only a few previous reports regarding the structure

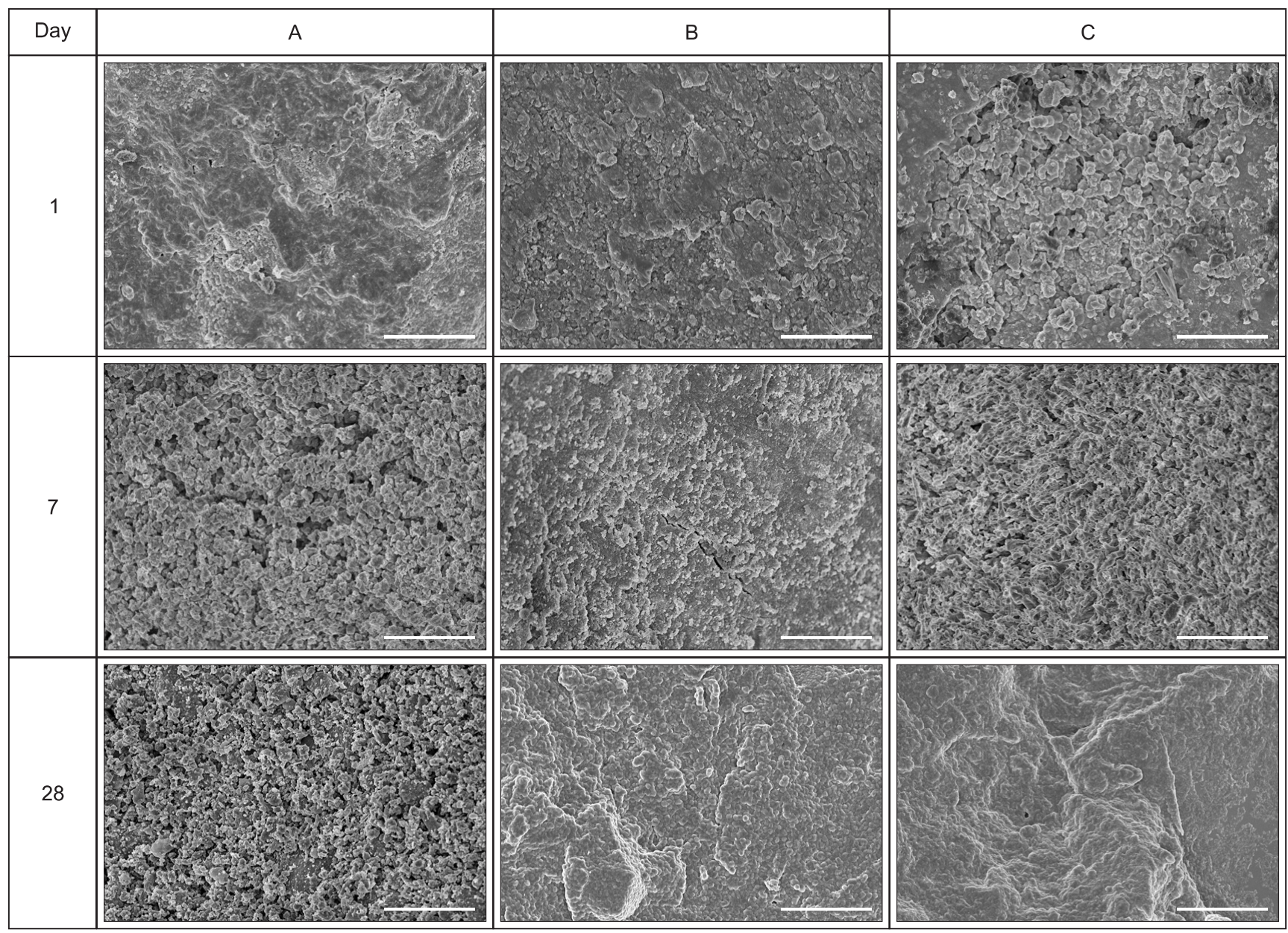

Fig. 5. Microstructure of the specimens observed by scanning electron microscopy. (A) Raw I, (B) Raw II, and (C) Raw III. Scale bars=10 $\mu$ m. 
and performance of alkali-activated BS/MK blends, and these are mainly focused on the MK-rich end of the continuum, where some BS is added to a metakaolin-based geopolymer to enhance strength development (Yip \& van Deventer, 2003; Yip et al., 2005; Buchwald et al., 2007; Yip et al., 2008).

In Raw III (MK:BS=0:100), the compressive strength was 10.83 MPa after 1 day and increased up to $48.25 \mathrm{MPa}$ after 28 days. The specimen mixed with BS showed higher compressive strength than the specimen with MK only. Because BS is a typical binding material that has strong reactivity, it participates not only in the geo-polymerization reaction but also in a Pozzolan reaction. Therefore, it particularly contributes to the long-term improvement of the compressive strength of the specimens. As shown in Fig. 4, the compressive strength of the Raw III specimen was as low as $10.83 \mathrm{MPa}$ at the early stage of curing (1 day); however, it increased drastically up to 48.25 $\mathrm{MPa}$ after a long curing time (28 days), which means that the typical Pozzolan reaction affects the final compressive strength of geo-polymers. This is also strong evidence of the above speculation.

When BS is mixed with MK at the same time, the improvement of compressive strength can be realized in both short-term and long-term curing periods. Moreover, it is very desirable from a recycling point of view to use greater amounts of BS, as it is normally a waste material. In particular, the composition of the Raw II specimen can be applied to fields that require high initial compressive strength because the compressive strength (26.41 MPa) after 1 day curing was relatively higher than the other samples.

As shown in Fig. 5, dense structures were found in Fig. 5B and $\mathrm{C}$ regardless of curing time; however, it seems that the structure became denser as curing time increased. On the other hand, many cracks and pores were found in Fig. 5A at all curing stages. In conclusion, the microstructure of the sample is in good agreement with the compressive strength data.

\section{Effect of Alkali Activator}

As shown in Table 3, the ratio of MK:BS was fixed at 50:50 and 0:100 in order to investigate the effect of alkali activator on compressive strength under high temperature pre-curing. Fig. 6 shows the results in a graph. As the BS powder contains a great deal of $\mathrm{CaO}(43.13 \mathrm{wt} \%)$ as shown in Table 1, it was expected that there would be a similar hydration reaction during geo-polymer formation as observed in ordinary cement, which would result in high compressive strength. Therefore, the effect of alkali activator(s) on the compressive strength of the geo-polymers was investigated.

As shown in Fig. 6, the compressive strength of the specimens with alkali activators was much higher than that of specimens without it. For the specimens without activator, cured up to 28 days, all compressive strength values were lower than the limit of detection as shown in Fig. 6. Contrary to our expectations, this result indicates that the Pozzolan reaction by large quantities of $\mathrm{CaO}$ in $\mathrm{BS}$ does not contribute to the geo-polymer formation at high temperature pre-curing. The compressive strength of the AA II specimen after 1 day curing was $52.01 \mathrm{MPa}$. The geo-polymer with strong alkali activator can elute $\mathrm{Si}^{4+}$ and $\mathrm{Al}^{3+}$ ions from the alumino-silicate compounds in the specimen. As the concentration of $\mathrm{OH}^{-}$is high, the $\mathrm{SiO}_{2}-\mathrm{Al}_{2} \mathrm{O}_{3}$ glassy bonding is quickly decomposed and large amounts of reactive ions are produced. Therefore, if the alkali ion density is high, the compressive strength would also be high and durable geo-polymer paste would be synthesized. The alkali activator is the important determinant of MK activation as it decomposes the MK reactant. The alkali activator helps the ion elution from $\mathrm{SiO}_{2}$ and $\mathrm{Al}_{2} \mathrm{O}_{3}$, which produces the reactive oxygen species that drive the geo-

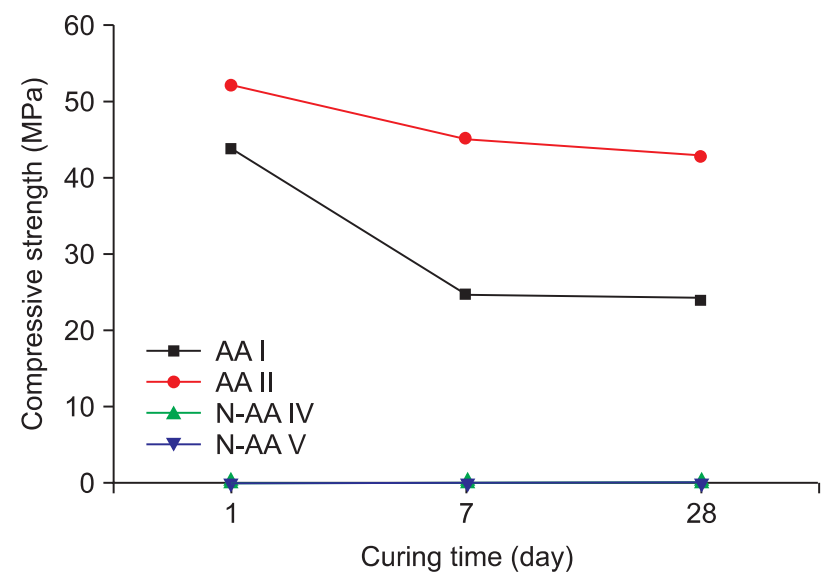

Fig. 6. Effect of alkali activators on the compressive strength of geopolymer specimens. AA I (MK:BS=50:50), AA II (MK:BS=0:100), N-AA III (MK:BS=50:50), and N-AA IV (MK:BS=0:100).

Table 3. Mixture of ratios of raw materials and alkali activators

\begin{tabular}{lccccc}
\hline Sample & MK:BS & $\mathrm{NaOH}(\mathrm{M})$ & Water glass (wt\%) & Water (wt\%) & Pre-curing condition \\
\hline AA I & $50: 50$ & 19.48 & 33.33 & 66.67 & $75^{\circ} \mathrm{C}, 6 \mathrm{hr}$ \\
AA II & $0: 100$ & - & - & - & - \\
N-AA III & $50: 50$ & 0 & 0 & 100 & - \\
N-AA IV & $0: 100$ & - & - & - & - \\
\hline
\end{tabular}


polymer reaction. Here, specimens without alkali activators did not show detectible values of compressive strength because the ion elution in the specimen was not realized.

As we can see from Fig. 6, the compressive strength of the AA I and AA II specimens decreased with increasing curing time. A very high compressive strength at an early stage of curing can be obtained with high temperature curing because the geopolymer reaction by alkali activators occurred very quickly as the BS content increased. On the other hand, microcracks caused by rapid volume expansion were generated by the hydration of $\mathrm{CaO}$ under high temperature pre-curing. These micro-cracks decreased the compressive strength with increasing curing time. In particular, there was a drastic decrease in compressive strength of AA I specimen with increasing curing time when MK and BS were mixed half and half. This drastic decrease in compressive strength, especially at the early stage of curing, can be explained by simultaneous occurrence of geo-polymerization and hydration reactions, resulting in undesirable interactions.

If we compare Fig. 4 and Fig. 6, the compressive strength of the specimen pre-cured at high temperature is higher than that of the specimen without pre-curing for the same ratio of MK and BS, especially at the early stage of curing. On the other hand, as curing time increased, the compressive strength of the specimen pre-cured at high temperature was about the same or even lower. In conclusion, the high temperature pre-curing plays an important role on the improvement of compressive strength of geo-polymers at the early stage; however, there was little effect or even a worsening effect on the compressive strength of geo-polymers with long-term curing.

\section{Effect of Pre-curing Condition}

\section{Effect of pre-curing time}

Fig. 7 shows the compressive strength with respect to three different pre-curing times $\left(3,6\right.$, and 24 hours) at $75^{\circ} \mathrm{C}$ before ordinary curing ( 1,7 , and 24 days) at room temperature. The fabrication conditions of the specimens are given in Table 4. As shown in Fig.7, compressive strength increased as curing time of the geo-polymer increased. There was little difference in compressive strength with respect to the different precuring times at high temperature before curing at room temperature. The geo-polymer specimens made only of MK did not show a decrease in compressive strength with longterm curing, which was different from the specimens mixed with BS. The best compressive strength (51.06 MPa) was obtained with 24 hours pre-curing at $75^{\circ} \mathrm{C}$ and 28 days curing at room temperature.

By comparing Fig. 4 and Fig. 7, it is notable that pre-curing at high temperature $\left(75^{\circ} \mathrm{C}\right)$ before ordinary curing is able to enhance the compressive strength of the geo-polymer, regardless of pre-curing time. Therefore, the pre-curing process at high temperature $\left(75^{\circ} \mathrm{C}\right)$ for 3 hours before ordinary curing is recommended for improving the mechanical properties of

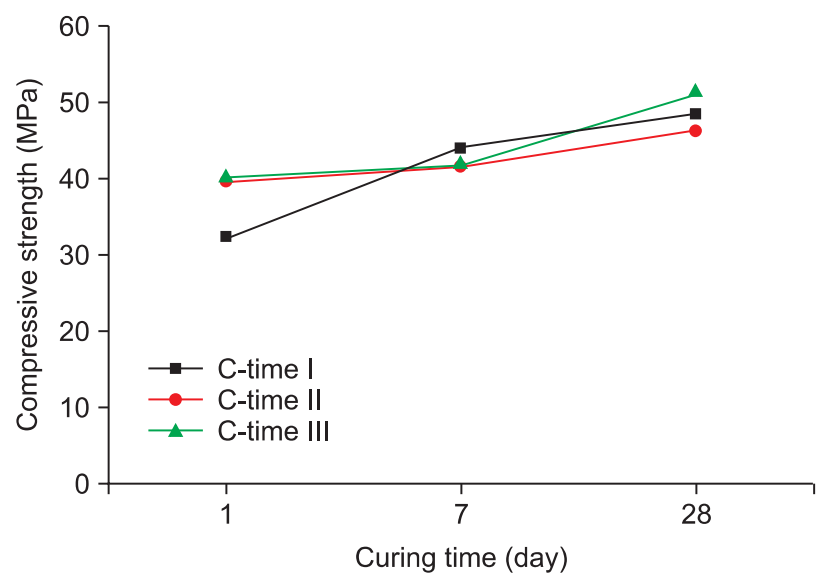

Fig. 7. Effect of pre-curing time on the compressive strength of geopolymer specimens. C-time I, pre-curing time 3 hours; C-time II, precuring time 6 hours; and C-time III, pre-curing time 24 hours.

Table 4. Mixture ratio of raw materials and alkali activators for the geo-polymer specimens with different pre-curing time at $75^{\circ} \mathrm{C}$ before ordinary curing at room temperature

\begin{tabular}{|c|c|c|c|c|c|c|c|c|}
\hline Sample & Pre-curing time (hr) & MK:BS & $\mathrm{NaOH}(\mathrm{M})$ & Liquid/solid & Water glass (wt\%) & Water (wt\%) & Pre-curing temperature $\left({ }^{\circ} \mathrm{C}\right)$ & Curing condition \\
\hline C-time I & 3 & 100:0 & 19.48 & 0.58 & 33.33 & 66.67 & 75 & 1,7 , and 28 days \\
\hline C-time II & 6 & - & - & - & - & - & - & - \\
\hline C-time III & 24 & - & - & - & - & - & - & - \\
\hline
\end{tabular}

Table 5. Mixture ratio of raw materials and alkali activators for the geo-polymer specimens with different pre-curing temperature

\begin{tabular}{lcccccc}
\hline \multicolumn{1}{c}{ Sample } & Pre-curing temperature $\left({ }^{\circ} \mathrm{C}\right)$ & MK:BS & NaOH $(\mathrm{M})$ & Water glass (wt\%) & Water $($ wt\%) & Pre-curing time $(\mathrm{hr})$ \\
\hline C-temperature I & Room & $0: 100$ & 19.48 & 33.33 & 66.67 & 24 \\
C-temperature II & 50 & - & - & - & - & - \\
C-temperature III & 75 & - & - & - & - & - \\
\hline
\end{tabular}


the geo-polymer when the specimens are made only of MK.

\section{Effect of pre-curing temperature}

Table 5 shows the fabrication conditions of the geo-polymer made of only BS. The reason for choosing BS-only specimens to investigate the effect of pre-curing temperature is due to the relatively larger volume expansion by hydration of $\mathrm{CaO}$ in BS compared to MK. Fig. 8 shows the compressive strength of the geo-polymer specimens with respect to the pre-curing temperature. Table 5 shows the conditions of specimen fabrication and pre-curing. As shown in Table 5, pre-curing was done for 24 hours at three different temperatures (room temperature, $50^{\circ} \mathrm{C}$ and $75^{\circ} \mathrm{C}$ ).

On the whole, compressive strength tends to increase as curing time increases. The result of C-temperature. III in Fig. 8 appears different from the result of AA II in Fig. 6; however, the absolute difference between these two results is small,

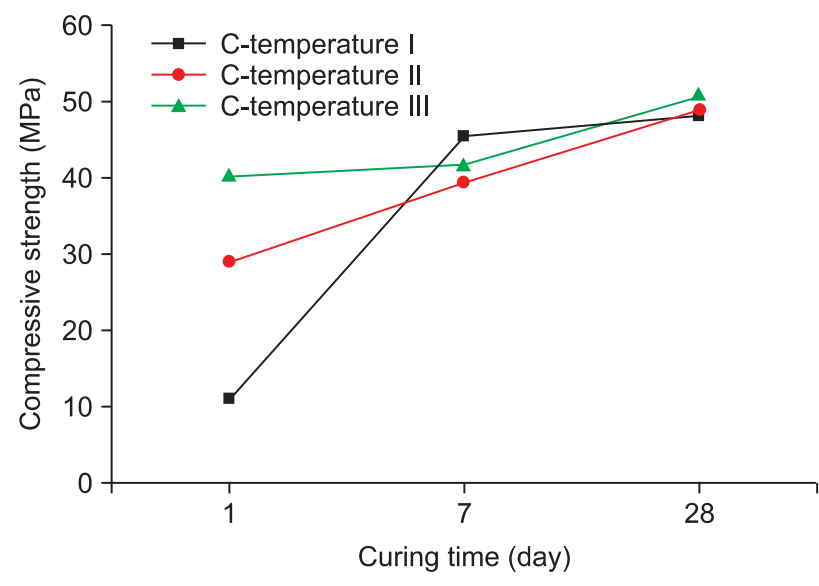

Fig. 8. Effect of pre-curing temperature on the compressive strength of geo-polymer specimens. C-temperature I, room temperature; C-temperature II, $50^{\circ} \mathrm{C}$; and C-temperature $\mathrm{III}, 75^{\circ} \mathrm{C}$.

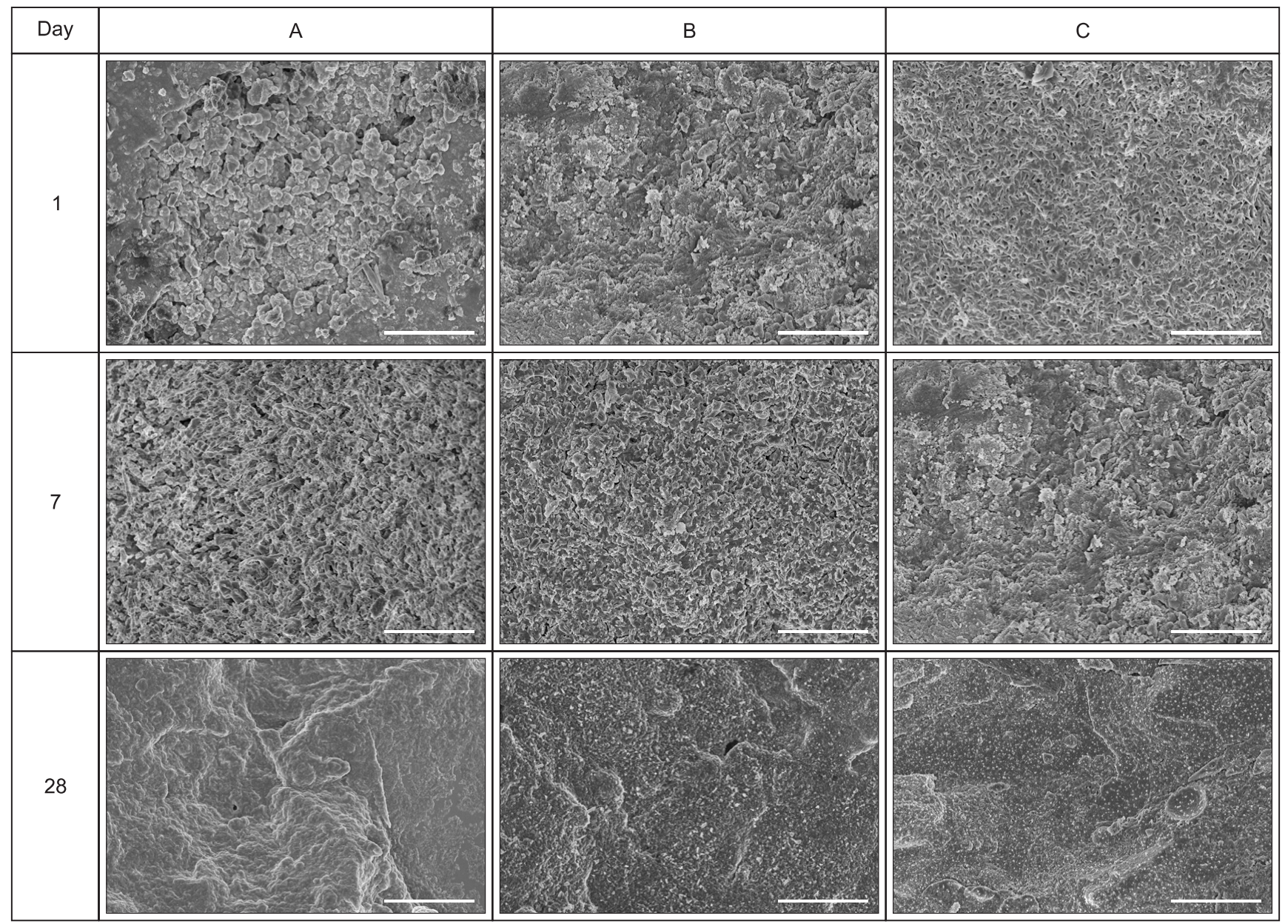

Fig. 9. Microstructure of the specimens observed by scanning electron microscopy. (A) C-temperature I, (B) C-temperature II, and (C) C-temperature III. Scale bars $=10 \mu \mathrm{m}$. 
which we can accept as within experimental error.

In particular, it is remarkable that a substantial improvement in compressive strength was found in the specimens made only of BS and pre-cured at high temperature, especially in the early stage of curing. For longer curing (up to 28 days), there was little change in compressive strength. In the case of room temperature curing, the compressive strength was poor $(10 \mathrm{MPa})$ in the early curing stage (1 day); however, it became equivalent to those of pre-cured specimens after long-term curing (after 7 days). This result is due to the Pozzolan reaction playing a role in the longterm compressive strength because of the large amount of $\mathrm{CaO}$ in the BS. For low temperature pre-curing, a decrease in compressive strength caused by volume expansion was not observed because expansion by the hydration of $\mathrm{CaO}$ in BS did not occur actively at lower temperatures (from room temperature to $50^{\circ} \mathrm{C}$ ). Therefore, the compressive strength can be continuously increased by medium temperature $\left(50^{\circ} \mathrm{C}\right)$ pre-curing without any decrease in compressive strength by volume expansion over the entire curing period.

In conclusion, high temperature pre-curing enhances the compressive strength in early stages but volume expansion can prevent it from further improvement in the long-term. On the other hand, a continuous increase in compressive strength over the entire curing period as well as a good compressive strength in the early stages can be realized by medium temperature $\left(50^{\circ} \mathrm{C}\right)$ pre-curing.

In Fig. 9, denser structures were found on the specimens pre-cured at higher temperature and at longer curing times. The micrograph in Fig. 9C (28 days) shows many microcrystallites in the matrix, which seem to be the main cause of compressive strength improvement in the geo-polymer cured at high temperature. No micro-cracks or voids were found in this micrograph unlike the case in the AA II specimen. Disagreement between the compressive strength trends in Fig. 6 (AA II) and Fig. 8 (C-temperature III) must be further investigated in a future study. In addition, more experiments on the compressive strength of the $\mathrm{MK}: \mathrm{BS}=50: 50$ specimens with respect to the curing temperature should be carried out and the volume expansion phenomenon by $\mathrm{CaO}$ hydration should also be explained in detail in a further study.

\section{CONCLUSIONS}

The compressive strength was enhanced for both short-term and long-term curing periods when BS was added to MK. In order to improve the recycling rate of BS and still obtain high compressive strength of geo-polymers, it is necessary to provide additional $\mathrm{CaO}$ to $\mathrm{MK}$ by adding $\mathrm{BS}$. The specimens containing greater amounts of BS, as in the Raw II sample, can be applied to fields such as emergency road repair in which high initial compressive strength is required.

No measurable compressive strength was observed for geopolymers without alkali activator, demonstrating that alkali activator(s) are required to make a useful geo-polymer. High temperature pre-curing plays an important role on the improvement of compressive strength in geo-polymers at the early stages; however, there was little effect or even a worsening effect on the compressive strength of geo-polymers with long term curing because of micro-structural defects by volume expansion of crystallites that were formed in the pre-curing period at high temperature. Therefore, a precuring process at medium-range is recommended because a continuous increase in compressive strength over the entire curing period as well as a good compressive strength in the early stages can be realized by medium temperature $\left(50^{\circ} \mathrm{C}\right)$ pre-curing.

Disagreement between the compressive strength trends in Fig. 6 and Fig. 8, the compressive strength of the MK:BS=50:50 specimens with respect to the curing temperature, and the volume expansion phenomenon by $\mathrm{CaO}$ hydration are recommended for future study.

\section{REFERENCES}

Bernal S A, Provis J L, Rose V, and De Gutierrez R M (2011) Evolution of binder structure in sodium silicate-activated slag-metakaolin blends. Cement \& Concrete Composites 33, 46-54.

Buchwald A, Hilbig H, and Kaps C (2007) Alkali-activated metakaolinslag blends--performance and structure in dependence on their composition. J. Mater. Sci. 42, 3024-3032.

Glasser F P (1996) Properties of cement waste composites. Waste Manage. 16, 159-168.

Komnitas K and Zaharaki D (2007) Geopolymerisation: a review and prospects for the minerals industry. Miner Eng. 20, 1261-1277.

Provis J L, Yong C Z, Duxson P, and van Deventer J S J (2009) Correlating mechanical and thermal properties of sodium silicate fly ash geopolymer. J. Mater. Sci. 336, 57-63.

Regourd M (1995) Cement Portland Hydration-hydraulic Concrete: Knowledge and Practice (ENPC, Paris).

Temuujin J and van Riessen A (2009) Effect of fly ash preliminary calcination on the properties of geopolymer. J. Harzard Mater. 164, 634-639.

Yip C K, Luckey G C, Provis J L, and van Deventer J S J (2008) Effect of calcium silicate sources on geopolymerisation. Cem. Concr. Res. $\mathbf{3 8}$, 554-564.

Yip C K, Lukey G C, and van Deventer J S J (2005) The coexistence of geopolymeric gel and calcium silicate hydrate at the early stage of alkaline activation. Cem. Concr. Res. 35, 1688-1697. 
Yip C K and van Deventer J S J (2003) Microanalysis of calcium silicate hydrate gel formed within a geopolymeric binder. J. Mater. Sci. $\mathbf{3 8}$, 3851-3960.

Zhao Q, Nair B, Rahimian T, and Balaguru P (2007) Novel geopolymer based composites with enhance ductility. J. Mater. Sci. 42, 3131-
3137.

Zhou J, Qian S, Beltran M G S, Ye G, van Breugel K, and Li V C (2010) Development of engineered cementitious composities with limestone powder and blast furnace slag. Mater. Struct. 43, 803-814. 